\title{
A Síndrome de Burnout no Contexto da ESF: Uma Análise das suas Dimensões
}

Livia de Sousa Rodrigues ${ }^{1}$; Silvia Morais de Santana ${ }^{2}$; Gislene Farias de Oliveira ${ }^{3}$

\begin{abstract}
Resumo: Introdução: Profissionais da Estratégia Saúde da Família, normalmente são submetidos a uma alta demanda técnico-científica, humana e emocional. Tal contexto tende a conduzir a fatores predisponentes ao adoecimento, com impacto sobre a qualidade da assistência prestada. Objetivo: Avaliar as dimensões da Síndrome de Burnout em profissionais da Estratégia Saúde da Família. Método: Estudo descritivo, transversal de abordagem quantitativa. Realizado em Várzea Alegre - CE, com 162 profissionais, participantes da ESF. O instrumento utilizado para coleta de dados foi o Inventário de Burnout de Maslach. Os dados foram analisados via pacote estatístico SPSS/Win, versão 18.0, com estatística descritiva. Resultados: A amostra contou com uma maioria de mulheres, casadas, católicas e com média de idade de 42 anos, predominantemente agentes comunitários de saúde. Foram detectados níveis baixos de Exaustão Emocional, em alguns casos com sinais de despersonalização. Considerações Finais: O trabalho na ESF submete os trabalhadores a um panorama de complexas e intensas relações interpessoais, o que pode produzir uma maior carga emocional. Urge a necessidade de medidas para a promoção de maior integração entre os atores envolvidos no processo de cuidado, com redução da pressão das relações interpessoais e da carga de trabalho sobre as emoções dos trabalhadores
\end{abstract}

Palavras-chave: Saúde da Família; Pessoal de Saúde; Síndrome de Burnout; Esgotamento profissional.

\section{Burnout Syndrome in the Context of ESF: An Analysis of its Dimensions}

\begin{abstract}
Professionals of the Family Health Strategy are usually submitted to a high technical-scientific, human and emotional demand. Such context tends to lead to factors predisposing to illness, with an impact on the quality of care provided. The aim is to evaluate the dimensions of Burnout Syndrome in professionals of the Family Health Strategy. Method: Descriptive, cross-sectional study of quantitative approach. Held in Várzea Alegre, Ceará, with 162 professionals, participants of the ESF. The instrument used for data collection was the Maslach Burnout Inventory. The data were analyzed using statistical package SPSS / Win, version 18.0, with descriptive statistics. Results: The sample had a majority of women, married, Catholic and with an average age of 42 years, predominantly community health agents. Low levels of Emotional Exhaustion were detected, in some cases with signs of depersonalization. Final Considerations: Work in the FHT subjects workers to a panorama of complex and intense interpersonal relationships, which can produce a greater emotional load. There is a need for measures to promote greater integration among the actors involved in the care process, reducing the pressure of interpersonal relationships and the workload on workers' emotions.
\end{abstract}

Keywords: Family Health; Health Staff; Burnout Syndrome; Professional Exhaustion.

\footnotetext{
${ }^{1}$ Mestre em Saúde da Família (RENASF/URCA); Enfermeira da Estratégia Saúde da Família em Várzea Alegre - Ceará. Contato: liviarodrigues_enf@hotmail.com;

${ }^{2}$ Mestranda em Ciências da Saúde pela FMABC/SP, Pós Graduação em Gestão de Pessoas (2008) e Graduada em Psicologia pela Faculdade do Rio Grande do Norte. Docente do curso Psicologia na Universidade Leão Sampaio, do curso de Pós Graduação em Psicologia Organizacional e do Trabalho da Unileão e da Pós Graduação em Gestão de Pessoas na Faculdade do Juazeiro do Norte.

Contato: silviamoraissf@gmail.com;

${ }^{3}$ Doutora em Psicologia Social pela Universidade Federal da Paraíba. Pós doutorado em Ciências da Saúde pela FMABC-SP. Docente da

Universidade Federal do cariri - UFCA. Contato: gislenefarias@gmail.com.
} 


\section{Introdução}

Avanços no campo da saúde coletiva brasileira vem ocorrendo nas últimas décadas, entretanto alguns fatores ainda dificultam a continuidade do processo de reorganização da Atenção Primária à Saúde (APS) (CAMPOS, FARIA; SANTOS, 2010).

A Estratégia Saúde da Família (ESF) emerge como modelo preferencial nesse contexto reorganizativo, uma vez que seus elementos fundamentais possibilitam uma alternativa para o alcance dos princípios e diretrizes do Sistema Único de Saúde (BRASIL, 2012).

Seu trabalho é realizado por meio de uma equipe multiprofissional composta por no mínimo médico, preferencialmente especialista em medicina de família e comunidade, enfermeiro, de preferência especialista em saúde da família; auxiliar e/ou técnico de enfermagem e agente comunitário de saúde (ACS). A equipe pode contar ainda com o agente de combate às endemias (ACE), cirurgião-dentista, de preferência especialista em saúde da família, e auxiliar ou técnico em saúde bucal (BRASIL, 2017).

Esta lógica organizativa dos modelos de atenção à saúde e da produção do cuidado exige diversas habilidades técnicas, sobretudo dos enfermeiros, cujos processos de trabalho requerem o desenvolvimento de estratégias, a previsão de acontecimentos futuros e o esforço intelectual para resoluções e superações (SHIMIZU; CARVALHO JUNIOR, 2012 apud LORENZ; GUIRARDELLO, 2014).

O caráter multiprofissional do trabalho em equipe traz peculiaridades próprias de uma atuação de profissionais com formações diversas em busca de um objetivo comum, o que gera um estressor e complicador do processo de trabalho da ESF, descrito como a complexidade presente na tecnologia das relações, em virtude da necessidade de respeito entre as pessoas com conhecimentos, habilidades e comportamentos próprios (VIDAL et al., 2014).

Considerando que os profissionais, membros da ESF, estão em contato direto com o usuário, objetivando uma atenção integral e equitativa, e que são operadores de um sistema de saúde público e universal, supõe-se a alta demanda técnico-científica, humana e emocional a que são submetidos diariamente. Enfrentam limitações de toda ordem, desde aquelas relacionadas à complexidade do trabalho em equipe até as de execução de ações para as quais, muitas vezes, não encontram viabilidade, mediante a crise que o sistema de saúde tem 
enfrentado nos últimos anos. Tal panorama conduz o trabalhador a sentimentos de insatisfação frente à realidade laboral vivenciada, com potencial gerador do seu adoecimento.

A Síndrome de Burnout consiste em uma síndrome tridimensional com dimensões independentes, embora relacionadas entre si, a saber: exaustão emocional, despersonalização e realização profissional reduzida (MASLACH; JACKSON; LEITER, 1996 apud LORENZ; GUIRARDELLO, 2014).

$\mathrm{Na}$ mesma perspectiva etiológica, figuram como fatores para o desenvolvimento da SB ou esgotamento profissional, a exaustão emocional provocada pela exposição crônica a estressores, a redução da satisfação no trabalho e as dificuldades para lidar com os pacientes de forma humanizada (BENEVIDES-PEREIRA apud MARTINS et al., 2014).

Os conceitos citados apontam para a origem da síndrome alusiva às condições a que os trabalhadores estão expostos durante o exercício laboral, o que a insere no rol de doenças relacionadas ao trabalho mediante sua associação à exposição aos fatores presentes no ambiente onde ele é realizado, com risco à saúde e à segurança do trabalhador (LORENZ; GUIRARDELLO, 2014).

Contudo, mesmo diante da predominância desta relação causal com as condições de trabalho, atualmente remete-se também à singularidade das pessoas, situação laboral e contexto de vida (CODO; VASQUES-MENEZES, 2000, apud FELICIANO; KOVACS; SARINHO, 2010).

No Brasil, estudos apontam a presença da síndrome em profissionais da saúde em hospitais e constatam índices de prevalência significativos, inclusive em profissionais da ESF (LIMA et al., 2014; TRINDADE, 2007 apud MARTINS et al., 2014; TAMAYO; TRÓCCOLI, 2002 apud FELICIANO; KOVACS; SARINHO, 2010).

Estudo realizado com enfermeiros brasileiros atuando em Atenção Primária à Saúde apresenta como desafio adicional ao trabalho destes profissionais, a complexidade da dinâmica de vida das comunidades, onde estes tendem a se envolver com os problemas dos pacientes devido à inserção geográfica dos serviços (ROSSI; SANTOS; PASSOS, 2010 apud HOLMES et al, 2014).

A mesma ideia é destacada em outro estudo ao mencionar que a inserção dos trabalhadores nesse modelo de atenção os expõe à realidade comunitária onde os recursos são escassos frente às demandas reais. Além do que, as falhas na rede de atenção afetam a resolutividade das ações (TRINDADE; LAUTERT, 2010). 
Considerando a relativa escassez de estudos realizados no Brasil acerca da Síndrome de Burnout e sua relação com o trabalho na ESF, evidencia-se uma lacuna a ser preenchida com abordagem da temática de maneira mais incisiva e direcionada.

Assim, nossa pesquisa objetiva avaliar as dimensões da Síndrome de Burnout em profissionais da Estratégia Saúde da Família.

\section{Método}

Trata-se de um estudo descritivo, de delineamento observacional transversal e abordagem quantitativa, desenvolvido no Município de Várzea Alegre, ao sul do Ceará, na região do cariri cearense, com uma população de 38.434 habitantes (BRASIL, 2010).

A rede municipal de serviços de saúde conta com 14 equipes de Saúde da Família EqSF distribuídas de maneira homogênea pelo território municipal.

Foram incluídos na amostra todos os profissionais de saúde que estavam atuando na Estratégia Saúde da Família do município há pelo menos um ano. Não foram considerados para o estudo os profissionais afastados das atividades por qualquer motivo, inclusive em desvio de função, lotados na Unidade Básica de Saúde da Família onde trabalha a pesquisadora (por questões éticas e para evitar conflitos de interesse) e aqueles que não devolveram os instrumentos de coleta de dados.

Ao final, a amostra foi composta por 11 enfermeiros, quatro médicos, três cirurgiões dentistas, 14 técnicos/ auxiliares de enfermagem, quatro técnicos/ auxiliares em saúde bucal e 54 agentes comunitários de saúde.

O instrumento de coleta de dados foi distribuído após uma rápida explanação a respeito do objeto e dos objetivos do estudo com ênfase na importância da participação voluntária de cada profissional para a representatividade dos resultados, além do anonimato dos dados colhidos.

Foi avaliado o nível das dimensões da Síndrome de Burnout (baixo, médio ou alto) na amostra pesquisada com a utilização do Inventário de Burnout de Maslach (MBI) constituído por 16 itens sobre sentimentos e atitudes do profissional, pontuadas numa escala do tipo Likert de 7 pontos que vai de zero (nunca) a seis (todo dia) (MASLACH ; JACKSON; LEITER, 1996). 
Este instrumento direciona uma abordagem psicossocial analisando o esgotamento profissional a partir de três dimensões independentes: Exaustão Emocional, quando ocorre perda de recursos emocionais para lidar com o trabalho; Despersonalização, implicando no desenvolvimento de atitudes negativas ou insensibilidade com aqueles que recebem o serviço prestado; (3) Falta de Realização Pessoal, com a tendência de avaliar o próprio trabalho de maneira negativa a partir de sentimentos como baixa autoestima profissional (MARTINS et al., 2014).

O período de coleta se estendeu pelo prazo de 30 dias, ao final do qual a pesquisadora realizou um novo contato, reforçando a relevância da participação do maior número possível de profissionais através da devolução dos questionários respondidos e dos respectivos TCLEs assinados.

O material resultante da coleta foi digitado no banco de dados eletrônico SPSS/win (versão 18.0), com o auxílio do qual se realizou o tratamento estatístico para posterior análise dos dados.

Considerou-se uma margem de erro de cerca cinco pontos percentuais com intervalo de confiança de $95 \%$.

O processo de análise utilizou a estatística descritiva com base em medidas como frequências, médias, desvio-padrão, dentre outros, assim como referenciais da literatura indexada em bases de dados e portais de acesso à informação.

O Projeto foi aprovado pelo Comitê de Ética em Pesquisa (CEP) da Universidade Regional do Cariri (URCA), obtendo parecer favorável de $\mathrm{n}^{\circ}$ 1.397.050.

O Gestor Municipal de Saúde de Várzea Alegre - CE, através da assinatura de um Termo de Consentimento Institucional, autorizou a realização da pesquisa com os profissionais de saúde, uma vez que os mesmos encontravam-se a serviço do município.

O Termo de Consentimento Livre e Esclarecido (TCLE) foi utilizado para todos os participantes da população, sendo considerados para a amostra, além da obediência aos critérios de inclusão e exclusão, apenas aqueles que devolveram o termo devidamente assinado junto com os instrumentos de coleta de dados preenchidos.

Foram considerados todos os princípios da Resolução no 466/2012, do Conselho Nacional de Saúde - CNS, que trata sobre os aspectos éticos de pesquisas envolvendo seres humanos. 


\section{Resultados}

A tabela 1 apresenta os achados da nossa pesquisa em relação às dimensões da Síndrome de Burnout (SB).

Tabela 1 - Dimensões da Síndrome de Burnout nos profissionais da amostra

\begin{tabular}{cccc}
\hline Nível de Burnout & Baixo & Médio & Alto \\
\hline Exaustão Emocional & $\underline{80,9 \%}$ & $16,9 \%$ & $2,2 \%$ \\
\hline Despersonalização & $43,2 \%$ & $\underline{46,6 \%}$ & $10,2 \%$ \\
\hline $\begin{array}{c}\text { Envolvimento } \\
\text { Pessoal no Trabalho }\end{array}$ & - & - & $\underline{100,0 \%}$ \\
\hline
\end{tabular}

Fonte: Dados da Pesquisa.

A utilização dos referidos níveis (alto, médio e baixo) considerou os parâmetros adotados pelo Grupo de Estudos e Pesquisas sobre Estresse e Burnout - GEPEB (BENEVIDES-PEREIRA, 2002). Os valores dos escores padronizados para cada dimensão da SB, de acordo com a literatura utilizada, são os seguintes: Exaustão emocional (16 a 25); Despersonalização (3 a 8); Realização profissional (32 a 42).

Evidenciou-se, neste estudo, baixos níveis de Exaustão Emocional (EE), níveis baixos a intermediários de Despersonalização (DP), e altos escores de Envolvimento Pessoal com o Trabalho (EPT), após inversão dos pontos. A partir daí, pode-se ter uma visão global da saúde mental destes profissionais, que já manifestam sinais de desgaste com possibilidade para o desenvolvimento da dimensão da Despersonalização.

\section{Discussão}

A Síndrome de Burnout (SB) apresenta-se como um transtorno tridimensional caracterizado por: Exaustão Emocional (EE), cursando com a sensação de ter chegado ao limite devido ao esgotamento motivado pelo estresse crônico; Despersonalização (DP), que diz respeito a modificações na personalidade do indivíduo, afetando o seu comportamento em 
relação aos demais (sejam eles colegas, pacientes ou usuários); e a terceira dimensão, a Realização Profissional Reduzida (ou inversamente, o Envolvimento Pessoal com o Trabalho - EPT), que cursa com sentimentos de baixa autoestima e insuficiência, baixa ou nenhuma realização com o trabalho, chegando muitas vezes a induzir o profissional a querer abandonar o emprego (BENEVIDES-PEREIRA, 2002).

Cada uma das dimensão pode ser avaliada individualmente com pontuação independente, caracterizando um maior ou menor nível de Burnout. A dimensão da Realização Profissional Reduzida pode ser invertida, denotando, em seus escores inversos, sensações de bem estar em relação ao trabalho e aos resultados do mesmo.

Uma contradição em relação aos achados da pesquisa diz respeito a uma elevação nos níveis de despersonalização contrapostos a baixos índices de exaustão emocional, já que a literatura demonstra que a Exaustão Emocional precede a Despersonalização (CARNEIRO, 2010). Fica evidente, portanto, o caráter multicausal da SB, onde cada dimensão pode sofrer influência de fatores múltiplos e diversos entre si.

Os baixos índices de Exaustão Emocional (EE) e altos índices de Envolvimento Pessoal com o Trabalho (EPT) podem ser justificados pela faixa etária predominante entre os indivíduos pesquisados, cuja média em anos foi de 42,23.

O tempo e as vivências proporcionam aos indivíduos o desenvolvimento de habilidades de enfrentamento e superação de desgastes. Estudos comprovam que indivíduos com idades inferiores aos 40 anos apresentam altos índices de desgaste emocional e despersonalização e baixos escores de realização profissional (GUIDO et al., 2012; TRINDADE; LAUTERT., 2010).

Assim, infere-se que os mais jovens e com pouca vivência estariam mais vulneráveis à Síndrome de Burnout, uma vez que a pouca idade dificulta o desenvolvimento de comportamentos e atitudes de proteção contra a Síndrome.

Frente a um panorama onde as relações humanas são preteridas em favor do uso de tecnologias de média e alta densidade, encontram-se os profissionais de alto contato desenvolvendo suas atividades laborais em ambientes ricos em relações humanas iminentes e imanentes (GOULART et al., 2010).

Uma pesquisa realizada com Agentes Comunitários de Saúde (ACS) de um município mineiro destacou que o estado de despersonalização agravou-se pela proximidade com os problemas vivenciados pelos usuários do serviço (BARROSO; GUERRA, 2013). 
O cenário representaria menor magnitude se o acometimento dos profissionais nesta dimensão não fosse tão danoso para seu relacionamento interpessoal com os colegas da equipe multiprofissional e com os próprios usuários do serviço.

A predominância dos ACSs na amostra reflete, assim como em outros estudos, que estes profissionais possuem como fator gerador de estresse o fato de representarem alvo de grandes expectativas por parte da equipe e da comunidade (MAIA; SILVA; MENDES, 2011). Devido ao seu vínculo de residência e convivência com a comunidade, aliam as pressões do trabalho com a vivência em ambientes insalubres, perigosos e profícuos ao adoecimento. Vários aspectos relacionados a esta categoria profissional têm sido negligenciados, entre eles a qualidade de vida e o impacto deste fator sobre o trabalho que realizam (BARROSO; GUERRA, 2013).

Os enfermeiros também figuram em estudos como profissionais acometidos na dimensão da Despersonalização, o que pode ser explicado por características próprias do trabalho na ESF, entretanto o adoecimento pode ser evitado quando são vislumbradas como necessárias as melhorias em relação à estrutura física e material, incremento de profissionais nas UBS e comprometimento dos mesmos, apoio institucional e da comunidade, redução de carga horária e iniciativas de cuidado aos profissionais (RÉUS et al., 2014).

Devido às relações de ajuda que estabelecem com outras pessoas em seu ambiente de trabalho, os enfermeiros estão em segundo lugar entre as profissões mais vitimadas pela SB (OLIVEIRA; PEREIRA, 2012).

Em relação aos trabalhadores de Saúde Bucal, estudo realizado com estes profissionais revela que, da amostra total, 32,26\% dos dentistas e 6,45\% dos Auxiliares de Consultório Dentário (ACD) apresentam a SB instalada. Explica-se que a baixa incidência da SB entre os ACD pode ser devida ao fato de os mesmos não realizarem um trabalho diretamente com a comunidade, já que trabalham predominantemente no interior dos consultórios, onde a maior responsabilidade ficaria a cargo dos profissionais de nível superior. No mesmo estudo apresentam a SB acomete $56,67 \%$ dos médicos investigados, seguidos por $26,67 \%$ das enfermeiras, 33,33\% dos Auxiliares de Enfermagem e 42,16\% dos Agentes Comunitários de Saúde (ALBUQUERQUE; MELO; ARAÚJO NETO, 2012).

A identidade profissional dos médicos apresenta uma resistência à flexibilização, embora esteja presente nestes trabalhadores o desejo tensionado de mudança. Mesmo com o desgaste e a insatisfação, a possibilidade de compartilhamento de problemas laborais com 
outros membros da equipe favorece ao enfrentamento das situações de crise (FELICIANO; KOVACS; SARINHO, 2011).

É sabido que o indivíduo no contexto laboral pode desenvolver sensações e atitudes positivas em relação ao trabalho executado, mas que também os problemas, o desinteresse e a insatisfação podem ocorrer (FRANÇA; FERRARI, 2012).

Assim, deve-se atentar para as questões relacionadas à saúde mental dos trabalhadores, considerando seu impacto negativo para a qualidade de vida da pessoa acometida, bem como seu efeito deletério para a qualidade do serviço prestado e consequentemente para a população que acessa o mesmo.

\section{Considerações Finais}

Nesta pesquisa, a amostra foi predominantemente feminina, casada e católica, o que demonstra um perfil tradicional dos profissionais ligados às atividades de cuidado. Este perfil pode implicar em jornada extra de trabalho no lar. A maioria dos pesquisados ocupa o cargo de agente comunitário de saúde, fato este corroborado por outros estudos.

$\mathrm{Na}$ avaliação das dimensões da Síndrome de Burnout, foram encontrados baixos níveis de exaustão emocional e, embora ainda bem comprometidos e envolvidos com seu trabalho, os profissionais já apresentam sinais de despersonalização, o que é bastante relevante quando se trata de profissões envolvidas com o cuidado humano. $\mathrm{O}$ fato pode ser justificado pelo perfil de meia idade da amostra, uma vez que os mais jovens apresentam uma maior dificuldade para lidar com o estresse e o desgaste laboral. O fato de os ACSs comporem a maioria da amostra também contribui para essa justificativa, já que são alvo de grande expectativa por parte da equipe e da comunidade, gerando uma maior pressão no trabalho.

O processo de trabalho na ESF submete os trabalhadores a um panorama de complexas e intensas relações interpessoais, além da formação de vínculo, seja com a equipe de trabalho e gestão, ou com os usuários dos serviços. Tais características podem produzir uma maior carga emocional, por lidar diretamente com pessoas e sua diversidade de experiências de vida. 
Ao mesmo tempo, tal estratégia apresenta novos arranjos organizacionais e processos de trabalho, ainda estranhos para muitos profissionais, o que eleva os níveis de estresse, pelo perfil de novidade e pela resistência que muitos trabalhadores apresentam ante os novos papéis assumidos pelos membros das diferentes categorias profissionais envolvidas.

Urge a necessidade de implementação de medidas capazes de promover uma maior integração entre os atores envolvidos no processo de cuidado, de modo a reduzir a pressão produzida pelas relações interpessoais e pela carga de trabalho sobre as emoções dos trabalhadores com a devida distribuição de responsabilidades também aos gestores e às pessoas sob cuidado.

Outros enfoques podem ser produzidos em novas pesquisas acerca de fatores causadores de sobrecarga emocional nos trabalhadores visando ao direcionamento de ações mais efetivas e eficazes voltadas a este grupo profissional.

\section{Referências}

ALBUQUERQUE, Francisco José Batista de; MELO, Cynthia de Freitas; ARAUJO NETO, João Lins de. Avaliação da síndrome de burnout em profissionais da Estratégia Saúde da Família da capital paraibana. Psicol. Reflex. Crit., Porto Alegre , v. 25, n. 3, p. 542-549, 2012

BARROSO, Sabrina Martins. GUERRA, Adriane de Rocha Pereira. Burnout e qualidade de vida de agentes comunitários de saúde de Caetanópolis (MG). Caderno de Saúde Coletiva, Rio de Janeiro, v. 21, n. 3, p. 338-345, 2013.

BENEVIDES-PEREIRA, Ana Maria T. [org.]. Burnout: quando o trabalho ameaça o bemestar do trabalhador. 1.ed. São Paulo: Casa do Psicólogo, 2002.

BRASIL. Ministério da Saúde. Gabinete do Ministro. Portaria no 2.436, de 21 de setembro de 2017. Aprova a Política Nacional de Atenção Básica, estabelecendo a revisão de diretrizes para a organização da Atenção Básica, no âmbito do Sistema Único de Saúde (SUS). Diário Oficial da República Federativa do Brasil, Brasília, DF, 24 out. 2011.

BRASIL. Ministério da Saúde. Portaria no 1.823, de 23 de agosto de 2012. Institui a Política Nacional de Saúde do Trabalhador e da Trabalhadora. Diário Oficial [da] República Federativa do Brasil, Brasília, 2012.

BRASIL. Programa das Nações Unidas para o Desenvolvimento. Atlas do Desenvolvimento Humano do Brasil. Caracterização do Território de Várzea Alegre - CE. 2010. Disponível 
em: http://www.atlasbrasil.org.br/2013/pt/perfil_m/varzea-alegre_ce. Acesso em: 14 de maio de 2015.

CAMPOS, F.C.C.; FARIA H. P.; SANTOS, M.A. .Planejamento e avaliação das ações em saúde. Curso de Especialização em Atenção Básica à Saúde da Família. 2ed. Belo Horizonte: NESCON/UFMG, 2010. Disponível em:<https://www.nescon.medicina.ufmg.br $>$. Acesso em $12 / 02 / 2015$.

CARNEIRO, Rúbia Mariano. Síndrome de Burnout: Um Desafio para o Trabalho do Docente Universitário. 2010. 86 f. (Dissertação de mestrado). Centro Universitário de Anápolis - UniEvangélica, Anápolis.

CONSELHO NACIONAL DE SAÚDE (Brasil). Resolução no. 466, de 12 de dezembro de 2012. Brasília, 2012 [citado 2014 Mar 11]. Disponível em: http://www.conselho.saude.gov.br/web_comissoes/conep/index.html. Acesso em 04 jan. 2015

FELICIANO, Katia Virginia de Oliveira; KOVACS, Maria Helena; SARINHO, Sílvia Wanick. Burnout entre médicos da Saúde da Família: os desafios da transformação do trabalho. Ciência e saúde coletiva, Rio de Janeiro, v. 16, n. 8, p. 3373-3382, Aug. 2011.

FELICIANO, Katia Virginia de Oliveira; KOVACS, Maria Helena; SARINHO, Silvia Wanick. Superposição de atribuições e autonomia técnica entre enfermeiras da Estratégia Saúde da Família. Revista de Saúde Pública, São Paulo , v. 44, n. 3, p. 520-527, June 2010 .

FRANCA, Flávia Maria de; FERRARI, Rogério. Síndrome de Burnout e os aspectos sóciodemográficos em profissionais de enfermagem. Acta paulista de enfermagem, São Paulo, v. 25, n. 5, p. 743-748, 2012 .

GOULART, Carolina Brito et al. Fatores predisponentes da síndrome de Burnout em trabalhadores de um hospital público de média complexidade. Revista Espaço para a Saúde. Londrina, v. 11, n. 2, p. 48-55, jun. 2010.

GUIDO, Laura de Azevedo et al . Síndrome de Burnout em residentes multiprofissionais de uma universidade pública. Revista da escola de enfermagem da USP, São Paulo, v. 46, n. 6, p. 1477-1483, Dec. 2012 .

HOLMES, Ericka Silva et al. Burnout syndrome in nurses acting in primary care: an impact on quality of life. J. res.: fundam. care. v.6, n.4, 2014, p.1384-1395.

LIMA, Letícia de et al . Satisfação e insatisfação no trabalho de profissionais de saúde da atenção básica. Esc. Anna Nery, Rio de Janeiro , v. 18, n. 1, p. 17-24, Mar. 2014.

LORENZ, Vera Regina; GUIRARDELLO, Edinêis de Brito. O ambiente da prática profissional e Burnout em enfermeiros na atenção básica. Revista Latino-Americana de Enfermagem, Ribeirão Preto, v. 22, n. 6, 2014, p. 926-933. 
MAIA, Leandro Dias de Godoy; SILVA, Nicácio Dieger; MENDES, Patrícia Helena Costa. Síndrome de Burnout em agentes comunitários de saúde: aspectos de sua formação e prática. Revista brasileira de saúde ocupacional, São Paulo, v. 36, n. 123, p. 93-102, June 2011.

MARTINS, Leonardo Fernandes et al . Esgotamento entre profissionais da Atenção Primária à Saúde. Ciência e saúde coletiva, Rio de Janeiro, v. 19, n. 12, p. 4739-4750, Dec. 2014 .

MASLACH, C., S.E., JACKSON \& LEITER, M. P. The Maslach Burnout Inventory Test manual. 3. ed. Palo Alto, CA. Consulting Psycologist Press, 1996.

OLIVEIRA, Vanessa. PEREIRA, Telmo. Ansiedade, depressão e burnout em enfermeiros: Impacto do trabalho por turnos. Rev. Enf. Ref., Coimbra, v. serIII, n. 7, p. 43-54, jul. 2012 .

RÉUS, Keterlee Mafalda da Silva. et al. A síndrome de burnout dos enfermeiros na ESF. Revista Inova Saúde, Criciúma, v.3, n.2, p.109-129, nov. 2014.

TRINDADE, Letícia de Lima; LAUTERT, Liana. Síndrome de Burnout entre os trabalhadores da Estratégia de Saúde da Família. Revista da escola de enfermagem da USP, São Paulo, v. 44, n. 2, p. 274-279, June 2010 .

VIDAL, Selma Vaz et al . Problemas bioéticos na Estratégia Saúde da Família: reflexões necessárias. Revista Bioética, Brasília , v. 22, n. 2, p. 347-357, Aug. 2014.

\section{Como citar este artigo (Formato ABNT):}

RODRIGUES, Lívia de S.; SANTANA, Silvia M. de; OLIVEIRA, Gislene F. de. A Síndrome de Burnout no Contexto da ESF: Uma Análise das suas Dimensões. Id on Line Revista Multidisciplinar e de Psicologia, 2018, vol.12, n.39, p.879-890. ISSN: 1981-1179.

Recebido: 31.01 .2018

Aceito: 01.02.2018 